\title{
Efficacy and safety of anlotinib in patients with advanced non-small cell lung cancer
}

\author{
Yuejiao Zhong ${ }^{1 \#}$, Qiang $\mathrm{Wei}^{2 \#}$, You $\mathrm{Lu}^{3}$, Xiuliang Tang ${ }^{2}$, Zhongqiu Wang ${ }^{4}$, Lingxiang Chen ${ }^{1}$ \\ ${ }^{1}$ Department of Medical Oncology, the Affiliated Cancer Hospital of Nanjing Medical University \& Jiangsu Cancer Hospital \& Jiangsu Institute of \\ Cancer Research, Nanjing, China; ${ }^{2}$ Department of Ultrasonography, the Affiliated Cancer Hospital of Nanjing Medical University \& Jiangsu Cancer \\ Hospital \& Jiangsu Institute of Cancer Research, Nanjing, China; ${ }^{3}$ Department of Intervention, the Affiliated Cancer Hospital of Nanjing Medical \\ University \& Jiangsu Cancer Hospital \& Jiangsu Institute of Cancer Research, Nanjing, China; ${ }^{4}$ Department of Chest Surgery, the Affiliated Cancer \\ Hospital of Nanjing Medical University \& Jiangsu Cancer Hospital \& Jiangsu Institute of Cancer Research, Nanjing, China \\ Contributions: (I) Conception and design: Y Zhong, Q Wei; (II) Administrative support: Z Wang; (III) Provision of study materials or patients: Y Lu; \\ (IV) Collection and assembly of data: L Chen; (V) Data analysis and interpretation: Y Zhong, X Tang; (VI) Manuscript writing: All authors; (VII) \\ Final approval of manuscript: All authors. \\ \#These authors contributed equally to this work. \\ Correspondence to: Lingxiang Chen. Department of Medical Oncology, the Affiliated Cancer Hospital of Nanjing Medical University \& Jiangsu \\ Cancer Hospital \& Jiangsu Institute of Cancer Research, Nanjing, China. Email: bg4wfc@126.com.
}

Background: Non-small cell lung cancer (NSCLC) is the most common type of lung cancer and its incidence seriously affects human health. The purpose of this study was to evaluate the efficacy and safety of anlotinib in patients with advanced NSCLC.

Methods: A retrospective study was conducted on 150 patients with advanced NSCLC who were treated with anlotinib and discontinued treatment after disease progression or intolerance due to adverse events. Progression-free survival (PFS) of advanced NSCLC patients served as an endpoint. Kaplan-Meier survival curves were applied to evaluate the short-term efficacy of anlotinib treatment in advanced NSCLC patients. Results: The median PFS of the whole 150-patient cohort was 5.0 months in (95\% CI: 4.00-5.95), 5.0 months (95\% CI: 3.0-6.00) in 90 patients with adenocarcinoma, and 4.5 months (95\% CI: 4.00-7.00) in 60 patients with squamous cell carcinoma (P=0.676). The PFS was 6.5 months (95\% CI: 4.00-8.80) and 4.5 months (95\% CI: 4.00-5.60) in the first-/second-line and $\geq$ third-line patients, respectively $(\mathrm{P}=0.315)$. Following the Eastern Cooperative Oncology Group performance status (ECOG PS) score, the median PFS of 95 patients with a PS score 0-1 was 5.5 months (95\% CI: 4.50-6.50), and the median PFS of 55 patients with a PS score ntswas 4.0 months (95\% CI: 3.00-5.00) (P=0.221). For the 49 patients in the combination group the median PFS was 7.0 months (95\% CI: 4.00-9.00), while that of the 101 patients in the anlotinibalone group was 4.0 months in (95\% CI: 2.80-5.50) $(\mathrm{P}=0.010)$. In a separate analysis of the combination group, the median PFS of anlotinib combined with chemotherapy, epidermal growth factor receptor-tyrosine kinase inhibitor (EGFR-TKI), and immunotherapy was 5.5 months (95\% CI: 4.00-9.00), 12.0 months (95\% CI: 6.00-12.00), and 6.5 months (95\% CI: 4.00-9.80), respectively $(\mathrm{P}=0.036)$.

Conclusions: Anlotinib exhibits good tolerance and performance in prolonging the PFS of patients and has considerable potential as a treatment for advanced NSCLC.

Keywords: Anlotinib; non-small cell lung cancer (NSCLC); efficacy; adverse event

Submitted Aug 21, 2020. Accepted for publication Oct 11, 2020.

doi: $10.21037 /$ jtd-20-2855

View this article at: http://dx.doi.org/10.21037/jtd-20-2855

(C) Journal of Thoracic Disease. All rights reserved. 


\section{Introduction}

The incidence and mortality of lung cancer rank first across the world $(1,2)$, with the relevant data indicating that nonsmall cell lung cancer (NSCLC) accounts for approximately $80-85 \%$ of lung cancer cases (3). Most cases of NSCLC are diagnosed at locally advanced or advanced stages, and thus only a minority of patients can be treated with surgery (4). Currently, medical treatments including chemotherapy, targeted therapy, and immunotherapy are still important means for locally advanced or advanced NSCLC. In recent years, driver gene-based molecular targeted precision therapy has revolutionized the treatment paradigm for patients with advanced NSCLC, and new anti-tumor drugs are continually being developed (5-8). Anlotinib, a novel small molecule multi-target tyrosine kinase inhibitor (TKI) that was independently developed in China, can effectively inhibit vascular endothelial growth factor receptor (VEGFR), platelet-derived growth factor receptor (PDGFR), fibroblast growth factor receptor (FGFR), and stem cell growth factor receptor (c-Kit). It has demonstrated anti-tumor angiogenesis and tumor cell growth inhibition activity with good safety (9). Based on the ALTER 0303 trial, the China Food and Drug Administration (CFDA) has approved anlotinib for third-line-and-beyond treatment of advanced NSCLC (10). However, currently, there are limited data from clinical studies of anlotinib in advanced NSCLC cases. In this study, we thus retrospectively collected 150 patients with advanced NSCLC diagnosed in our hospital as the study subjects to evaluate the shortterm efficacy and safety of anlotinib in treating advanced NSCLC.

We present the following article in accordance with the STROBE reporting checklist (available at http://dx.doi. org/10.21037/jtd-20-2855).

\section{Methods}

\section{General data}

A total of 150 patients diagnosed with advanced NSCLC in Jiangsu Cancer Hospital were selected from June 2018 to August 2019. The general data of the patients were collected, including age, gender, age, pathological type, number of treatment lines, clinical stage, and Eastern Cooperative Oncology Group performance status (ECOG PS). The study was conducted in accordance with the Declaration of Helsinki (as revised in 2013). This study was approved by the Ethics Committee of Jiangsu Cancer Hospital, and all patients had signed informed consent.

\section{Inclusion criteria}

The inclusion criteria for patients were the following: (I) age $\geq 18$ years; and (II) advanced NSCLC, according to the American Foint Committee on Cancer Staging Manual, 8th edition staging, as divided into IIIB, IIIC, or IV.

\section{Exclusion criteria}

The exclusion criteria for patients were the following: (I) central lung squamous cell carcinoma, (II) history of hemoptysis, (III) abnormal coagulation function or bleeding tendency, (IV) uncontrolled hypertension, (V) thrombolysis or under an anticoagulant drug regime, or (VI) contraindication to drug treatment.

\section{Therapeutic approaches}

All NSCLC patients were treated with anlotinib (Fucovir, Chia Tai Tianqing Pharmaceutical Group, Sinopharm; batch no: H20180004), $12 \mathrm{mg} / \mathrm{d}$ orally for 2 weeks, followed by a 1 -week break, repeated every 3 weeks. If the disease could be controlled and the adverse events were tolerable, the patient continued to take the drug; if the patient presented with adverse events $\geq$ grade III, the dose of the drug was reduced; if the diseased progressed or intolerance due to adverse events emerged, the drug treatment was discontinued.

\section{Efficacy evaluation}

Efficacy was evaluated by computed tomography (CT) and magnetic resonance imaging (MRI) under Response Evaluation Criteria in Solid Tumors (RECIST, version 1.1) every 2 cycles after medication or when clinical symptoms worsened. The primary endpoint was progression-free survival (PFS), or the period from the start of anlotinib administration to tumor progression or death.

\section{Adverse events}

The safety of Anlotinib treatment was assessed using the Common Terminology Criteria for Adverse Events (CTCAE, version 4.0). 
Table 1 General data of advanced NSCLC patients

\begin{tabular}{lc}
\hline General data & Patients $(\mathrm{n}=150)(\%)$ \\
\hline Sex & $94(62.7)$ \\
M & $56(37.3)$ \\
Age (years) & \\
$\leq 65$ & $97(64.6)$ \\
$>65$ & $53(43.3)$ \\
Pathological type & \\
Adenocarcinoma & $90(60.0)$ \\
Squamous cell carcinoma & $60(40.0)$ \\
Number of treatment lines & \\
$<3$ & $25(16.7)$ \\
$\geq 3$ & $125(83.3)$ \\
Clinical phase & \\
IIIB/IIIC & $18(12.0)$ \\
IV & $132(88.0)$ \\
ECOG PS & \\
$0-1$ & $95(63.3)$ \\
\hline N & $55.7)$ \\
\hline
\end{tabular}

NSCLC, non-small cell lung cancer; ECOG PS, Eastern Cooperative Oncology Group Performance Status.

\section{Statistical methods}

SPSS 22.0 software was used for statistical analysis, and enumeration data are expressed as rate or constituent ratio. The PFS between the groups was analyzed using KaplanMeier survival curve. A P value $<0.05$ was considered statistically significant.

\section{Results}

\section{Patients enrolled and clinical characteristics}

Between June 2018 and August 2019, a total of 150 patients with advanced NSCLC were included, 94 of whom (62.7\%) were male and 56 of whom (37.3\%) were female. Demographic and clinical characteristics of patients were collected, including gender, age, pathological type, number of treatment lines, clinical stage, and ECOG PS score (Table 1).

\section{Short-term efficacy}

Kaplan-Meier survival analysis indicated that the median PFS of the 150 patients with advanced NSCLC treated with anlotinib was 5.0 months (95\% CI: 4.00-5.95), which was slightly lower than the 5.37 months reported in the ALTER0303 study (Figure 1A). According to pathology type, the PFS for the 90 patients with adenocarcinoma was 5.0 months (95\% CI: 3.00-6.00) and 4.5 months for the 60 patients with squamous cell carcinoma (95\% CI: 4.00-7.00) $(\mathrm{P}=0.676)$ (Figure 1B). PFS was 6.5 months in first- and second-line patients (95\% CI: 4.00-8.80), and 4.5 months for third-line-or-beyond (95\% CI: 4.00-5.60) $(\mathrm{P}=0.315)$ (Figure 1C). For ECOG PS scoring, 95 patients with a PS score of $0-1$ had a PFS of 5.5 months (95\% CI: 4.50-6.50), and 55 patients with a PS score $\geq 2$ had a PFS of 4.0 months (95\% CI: 3.00-5.00) $(\mathrm{P}=0.221)$ (Figure 1D). The median PFS of 49 patients in the combined treatment group [combined chemotherapy, combined immunosuppressive agent, combined epidermal growth factor receptor-tyrosine kinase inhibitor (EGFR-TKI)] was 7.0 months (95\% CI: 4.0-9.00), While the median PFS of 101 patients taking Anlotinib alone was 4.0 months (95\% CI: 2.80-5.50) $(\mathrm{P}=0.010)$ (Figure 1E). In a separate analysis of the combination arm, the PFS was 5.5 months in the chemotherapy combined treatment group (95\% CI: 4.00-9.00), 12.0 months in the combined EGFR-TKI group (95\% CI: 6.00-12.00), and 6.5 months in the combined immunization group (95\% CI: 4.00-9.80) ( $\mathrm{P}=0.036)$ (Figure 1F).

\section{Safety}

The adverse events which occurred in the 150 patients with advanced NSCLC treated with anlotinib were asthenia (18.0\%), anorexia symptoms (6.67\%), diarrhea (4.67\%), oropharyngeal pain $(10.0 \%)$, oral mucositis $(8.0 \%)$, vomiting $(2.0 \%)$, hemoptysis $(2.0 \%)$, hoarseness $(10.7 \%)$, hypertension $(50.7 \%)$, hand-foot-skin reaction $(16.0 \%)$, proteinuria (8.0\%), and unknown events (5.3\%) (Table 2).

\section{Discussion}

The treatment of NSCLC is a major challenge in modern medicine. At present, the drug treatment for patients with advanced NSCLC mainly includes chemotherapy, immunotherapy, and targeted therapy. The toxicity and 
A

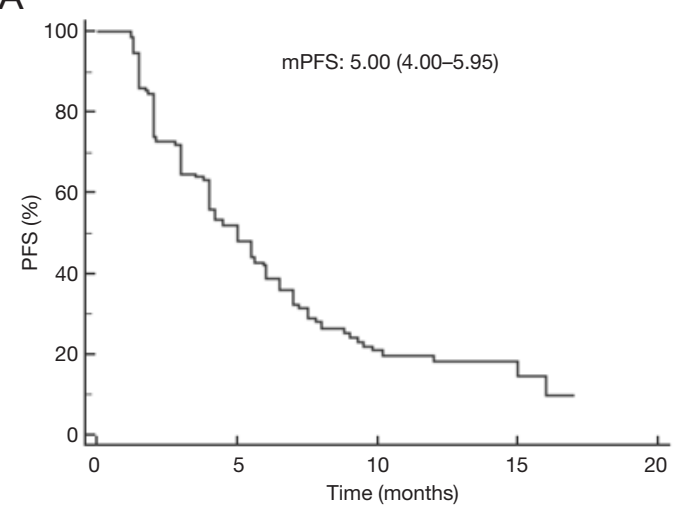

C

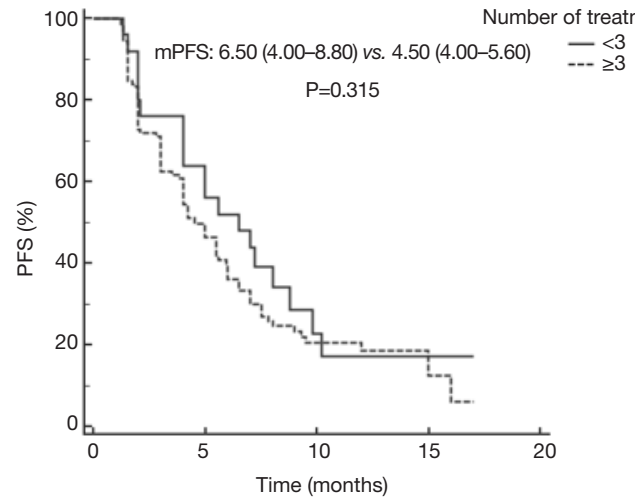

$\mathrm{E}$

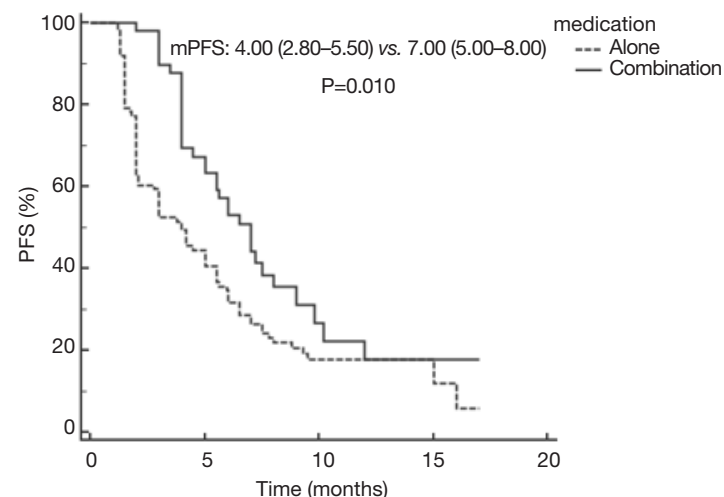

Figure 1 Efficacy observation after administration of anlotinib.

adverse events of chemotherapy for advanced NSCLC have limited its clinical application, but the emergence of targeted drug therapy has provided the possibility to prolong the survival time of patients. In recent years, the clinical study of anlotinib in the treatment of NSCLC has become an intensely researched subject (11-15). Anlotinib, as a novel small-molecule TKI, has multiple targets of action, which can effectively inhibit tyrosine
B

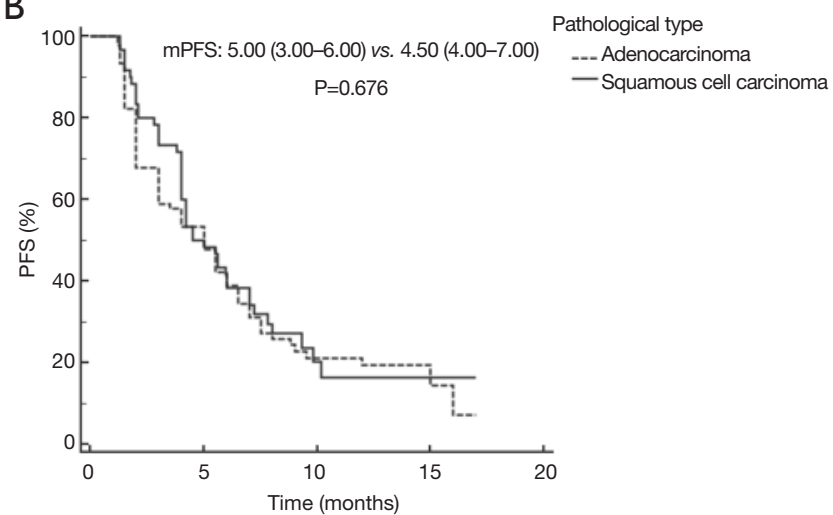

D

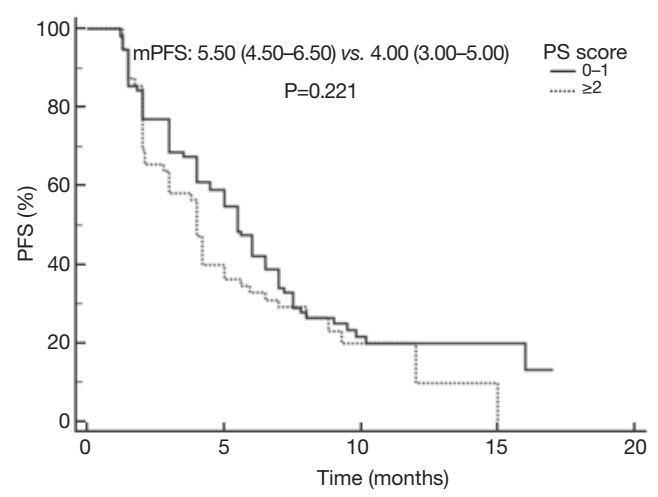

$\mathrm{F}$

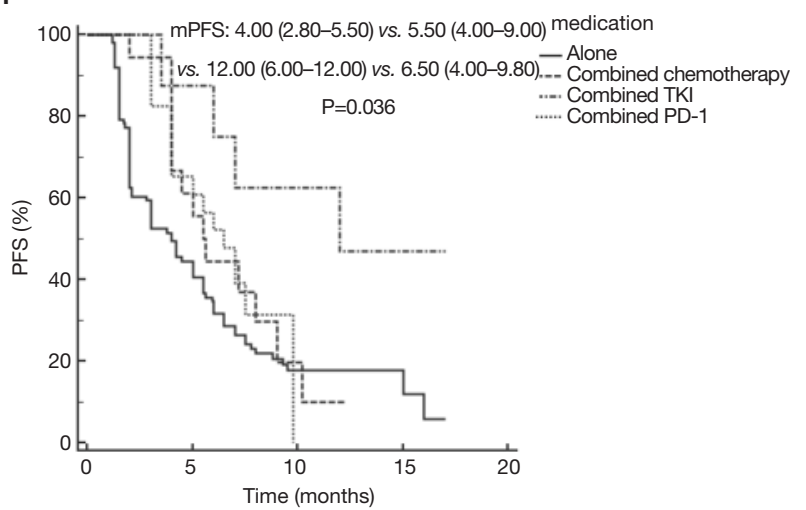

kinase activity, block EGFR-mediated tumor cell signaling, suppress angiogenesis, and inhibit tumor cell growth, thus promoting tumor cell apoptosis (16). Studies have revealed an advantage of anlotinib against tumors, whose inactivation effect for fibroblast growth factor receptor 1 (FGFR1), FGFR2, FGFR3, and FGFR4 is significantly better than that of sorafenib (9). Anlotinib has also shown the ability to constrain VEGFR2 at an approximate magnitude 20- 
Table 2 Incidence of adverse events of late-stage NSCLC patients treated with anlotinib

\begin{tabular}{lc}
\hline Adverse events & Patients, $\mathrm{n}(\%)$ \\
\hline General condition & $27(18.0)$ \\
Asthenia & $10(6.67)$ \\
Anorexia symptoms & \\
Gastrointestinal system & $7(4.67)$ \\
Diarrhea & $15(10.0)$ \\
Oropharyngeal pain & $12(8.0)$ \\
Oral mucositis & $3(2.0)$ \\
Vomiting & $3(2.0)$ \\
Hemoptysis & \\
Respiratory system & $16(10.7)$ \\
Hoarseness & \\
Cardiovascular system & \\
Hypertension & $76(50.7)$ \\
Skin system & \\
Hand-foot skin reaction & \\
Urinary system & $24(16.0)$ \\
Proteinuria & \\
\hline
\end{tabular}

NSCLC, non-small cell lung cancer.

and 500-fold higher than that of sunitinib and sorafenib, respectively $(17,18)$, and can effectively block c-Kit-related signaling pathways by inhibiting phosphorylation of kinases, such as ERK and protein kinase B (Akt) (18). It has been clinically found that the action concentration of anlotinib is low, but this can be solved by using a once-daily oral administration that can be easily performed, is safe, and can increase patient tolerance (19). In this study, 150 patients with advanced NSCLC were treated with anlotinib, and the efficacy and safety of the treatment were assessed, with the primary observation endpoint being PFS. The results showed that anlotinib had a marked effect on the clinical treatment of advanced NSCLC and could effectively prolong the PFS of patients, with good tolerability and safety.

Furthermore, the PFS of the 150 patients with advanced NSCLC treated with anlotinib was 5.0 months, which was slightly lower than the 5.37 months reported in the ALTER0303 study. This discrepancy may be related to the small sample size, low ECOG PS score in some patients, or the more than three lines of treatment involved. The results of subgroup analysis showed that PFS were not significantly different across patients with adenocarcinoma and squamous cell carcinoma, indicating that the efficacy of anlotinib in improving PFS was similar in patients with adenocarcinoma and squamous cell carcinoma. There was no statistically significant difference in PFS after oral anlotinib treatment between first-, second-, and third-lineand-beyond patients with advanced NSCLC, but first- and second-line treatment tended to prolong PFS in patients. According to ECOG PS score, PFS was 5.5 months for the 95 patients with a PS scored $0-1$ and 4.0 months for the 55 patients with a PS score $\geq 2$, with no significant difference.

We also compared the efficacy of anlotinib and anlotinib combined with other drugs in the treatment of advanced NSCLC, and the results were statistically different. The median PFS of 101 patients treated with Anlotinib alone was 4.0 months, and the median PFS of 49 patients treated with Anlotinib was 7.0 months, indicating that the combined use of Anlotinib is more effective. The PFS of the EGFR-TKI combination group, combination chemotherapy group, and combination immunization group were 12.0, 5.5, and 6.5 months, respectively, suggesting that the combination of anlotinib and EGFR-TKI can significantly prolong the PFS of patients with advanced NSCLC, and can be expected to be widely used in clinical practice.

The main adverse events in this study were fatigue, anorexia symptoms, diarrhea, oropharyngeal pain, oral mucositis, vomiting, hemoptysis, hoarseness, hypertension, hand-foot-skin reactions, and proteinuria, whose incidences were $18.0 \%, 6.67 \%, 4.67 \%, 10.0 \%, 8.0 \%, 2.0 \%, 2.0 \%$, $10.7 \%, 50.7 \%, 16.0 \%$, and $8.0 \%$, respectively. It was observed that most adverse events were mild and could be relieved by symptomatic treatment or adjustment of the administered dose.

In summary, anlotinib demonstrated a marked effect on the clinical treatment of advanced NSCLC patients; it could effectively prolong the PFS of patients, was well tolerated and safe, and may provide a new treatment option for patients with advanced NSCLC. Due to the small sample size of this study, future large-sample, prospective clinical trial studies are needed to further evaluate the efficacy and safety of anlotinib in treating advanced NSCLC.

\section{Acknowledgments}

Funding: The work was supported by the Talents Program 
of Jiangsu Cancer Hospital; Jiangsu Provincial Medical Youth Talent, The Project of Invigorating Health Care through Science, Technology and Education, Heath-pilot Cancer Research Fund (Y-2019AZMS-0556).

\section{Footnote}

Reporting Checklist: The authors have completed the STROBE reporting checklist. Available at http://dx.doi. org/10.21037/jtd-20-2855

Data Sharing Statement: Available at http://dx.doi. org/10.21037/jtd-20-2855

Conflicts of Interest: All authors have completed the ICMJE uniform disclosure form (available at http://dx.doi. org/10.21037/jtd-20-2855). The authors have no conflicts of interest to declare.

Etbical Statement: The authors are accountable for all aspects of the work in ensuring that questions related to the accuracy or integrity of any part of the work are appropriately investigated and resolved. The study was conducted in accordance with the Declaration of Helsinki (as revised in 2013). This study was approved by the Ethics Committee of Jiangsu Cancer Hospital, and all patients had signed informed consent.

Open Access Statement: This is an Open Access article distributed in accordance with the Creative Commons Attribution-NonCommercial-NoDerivs 4.0 International License (CC BY-NC-ND 4.0), which permits the noncommercial replication and distribution of the article with the strict proviso that no changes or edits are made and the original work is properly cited (including links to both the formal publication through the relevant DOI and the license). See: https://creativecommons.org/licenses/by-nc-nd/4.0/.

\section{References}

1. Chen W, Zheng R, Baade PD, et al. Cancer Statistics in China, 2015. CA Cancer J Clin 2016;66:115-32.

2. Sun M, Guo Y, Shao G, et al. Analysis of real-word mutations of lung cancer driver genes in five regions of China. Transl Cancer Res 2019;8:2581-92.

3. Molina JR, Yang PG, Cassivi SD, et al. Non-small cell lung cancer: Epidemiology, risk factors, treatment, and survivorship. Mayo Clin Proc 2008;83:584-94.
4. Filippou D, Kleontas A, Tentzeris V, et al. Extended resections for the treatment of patients with T4 stage IIIA non-small cell lung cancer (NSCLC) ( $\mathrm{T}(4) \mathrm{N}(0-1) \mathrm{M}(0))$ with or without cardiopulmonary bypass: a 15 -year twocenter experience. J Thorac Dis 2019;11:5489-501.

5. Spigel DR, Burris HA, Greco FA, et al. Erlotinib Plus Either Pazopanib or Placebo in Patients With Previously Treated Advanced Non-Small Cell Lung Cancer: A Randomized, Placebo-Controlled Phase 2 Trial With Correlated Serum Proteomic Signatures. Cancer 2018;124:2355-64.

6. Zhang H. Apatinib for molecular targeted therapy in tumor. Drug Des Devel Ther 2015;9:6075-81.

7. Lv S, Tang Z, Li M, et al. Co-delivery of doxorubicin and paclitaxel by PEG-polypeptide nanovehicle for the treatment of non-small cell lung cancer. Biomaterials 2014;35:6118-29.

8. Zhou C, Wu YL, Chen G, et al. Erlotinib versus chemotherapy as first-line treatment for patients with advanced EGFR mutation-positive non-small-cell lung cancer (OPTIMAL, CTONG-0802): a multicentre, open-label, randomised, phase 3 study. Lancet Oncol 2011;12:735-42.

9. Sun Y, Niu W, Du F, et al. Safety, pharmacokinetics, and antitumor properties of anlotinib, an oral multitarget tyrosine kinase inhibitor, in patients with advanced refractory solid tumors. J Hematol Oncol 2016;9:105.

10. Chinese Oncologist Association, Expert Committee on Vascular Targeted Therapy of Chinese Society of Clinical Oncology, Professional Committee on Tumor Targeted Therapy of Chinese Anti-Cancer Association: Expert Consensus on Anlotinib Hydrochloride in Treatment of Advanced Cell Lung Cancer. Chinese Medical Journal 2018;98:3561-7.

11. Zhang Z, Zhao Y, Lu F, et al. Multi-targeted tyrosine kinase inhibitors as third-line regimen in advanced nonsmall cell lung cancer: a network meta-analysis. Ann Transl Med 2019;7:452.

12. Wang $\mathrm{L}, \mathrm{He} Z$, Yang $\mathrm{S}$, et al. The impact of previous therapy strategy on the efficiency of anlotinib hydrochloride as a third-line treatment on patients with advanced non-small cell lung cancer (NSCLC): a subgroup analysis of ALTER0303 trial. Transl Lung Cancer Res 2019;8:575-83.

13. Chen XZ. Anlotinib for refractory advanced non-small cell lung cancer in China. JAMA Oncol 2019;5:116-7.

14. Yang S, Wang L, Wang Q. Previous Therapy Strategy Impact on Efficiency of Anlotinib Hydrochloride as 3rd 
Line Treatment: A Subgroup Analysis of ALTER0303

Trial. J Thorac Oncol 2019;14:S576.

15. Han B, Li K, Wang QM, et al. Effect of Anlotinib as a Third-Line or Further Treatment on Overall Survival of Patients With Advanced Non-Small Cell Lung Cancer The ALTER 0303 Phase 3 Randomized Clinical Trial. JAMA Oncol 2018;4:1569-75.

16. Hall RD, Le TM, Haggstrom DE, et al. Angiogenesis inhibition as a therapeutic strategy in non-small cell lung cancer (NSCLC). Transl Lung Cancer Res 2015;4:515-23.

17. Xie C, Wan X, Quan H, et al. Preclinical characterization

Cite this article as: Zhong Y, Wei Q, Lu Y, Tang X, Wang Z, Chen L. Efficacy and safety of anlotinib in patients with advanced non-small cell lung cancer. J Thorac Dis 2020;12(10):6016-6022. doi: 10.21037/jtd-20-2855 of anlotinib, a highly potent and selective vascular endothelial growth factor receptor-2 inhibitor. Cancer Sci 2018;109:1207-19.

18. Lin B, Song X, Yang D, et al. Anlotinib inhibits angiogenesis via suppressing the activation of VEGFR2, PDGFR $\beta$ and FGFR1. Gene 2018;654:77-86.

19. Xin T, Jin F, Liu W, et al. Clinical study of Anlotinib Hydrochloride Capsules in the treatment of advanced breast cancer. Chinese Journal of Pulmonary Diseases (Electronic Edition) 2018;11:559-62. 